\title{
Cathepsin $\mathrm{S}$ inhibitor prevents autoantigen presentation and autoimmunity
}

\author{
Kaoru Saegusa, ${ }^{1}$ Naozumi Ishimaru, ${ }^{1}$ Kumiko Yanagi, ${ }^{1}$ Rieko Arakaki, ${ }^{1}$ Kouichi Ogawa, ${ }^{1}$ \\ Ichiro Saito, ${ }^{1}$ Nobuhiko Katunuma, ${ }^{2}$ and Yoshio Hayashi ${ }^{1}$
}

${ }^{1}$ Department of Pathology, Tokushima University School of Dentistry, Tokushima, Japan

${ }^{2}$ Institute for Health Science, Tokushima Bunri University, Yamashirocho, Tokushima, Japan

\begin{abstract}
The cysteine endoprotease cathepsin S mediates degradation of the MHC class II invariant chain Ii in human and mouse antigen-presenting cells. Studies described here examine the functional significance of cathepsin $S$ inhibition on autoantigen presentation and organ-specific autoimmune diseases in a murine model for Sjögren syndrome. Specific inhibitor of cathepsin S (Clik60) in vitro markedly impaired presentation of an organ-specific autoantigen, $120-\mathrm{kDa} \alpha$-fodrin, by interfering with MHC class II-peptide binding. Autoantigen-specific $\mathrm{T}$ cell responses were significantly and dose-dependently inhibited by incubation with Clik60, but not with inhibitor s of cathepsin B or L. Clik60 treatment of mouse salivary gland cells selectively inhibited autopeptide-bound class II molecules. Moreover, the treatment with Clik60 in vivo profoundly blocked lymphocytic infiltration into the salivary and lacrimal glands, abrogated a rise in serum autoantibody production, and led to recovery from autoimmune manifestations. Thus, inhibition of cathepsin $S$ in vivo alters autoantigen presentation and development of organ-specific autoimmunity. These data identify selective inhibition of cysteine protease cathepsin $\mathrm{S}$ as a potential therapeutic strategy for autoimmune disease processes.
\end{abstract}

J. Clin. Invest. 110:361-369 (2002). doi:10.1172/JCI200214682.

\section{Introduction}

Sjögren syndrome (SS) is an autoimmune disorder characterized by lymphocytic infiltrates and destruction of the salivary and lacrimal glands, and systemic production of autoantibodies to the ribonucleoprotein particles SS-A/Ro and SS-B/La (1-3). We have investigated an animal model for SS in NFS/sld mutant mice thymectomized 3 days after birth (3d-Tx) (4-9). All $3 \mathrm{~d}-\mathrm{Tx} \mathrm{NFS} /$ sld mice develop autoimmune lesions in the salivary and lacrimal glands, starting at 3 weeks of age, and the disease mediated by $\mathrm{CD} 4^{+} \mathrm{T}$ cells is chronic and progressive $(4,5)$. Previously, we reported a $120-\mathrm{kDa}$ $\alpha$-fodrin autoantigen in the salivary gland tissues from SS model mice and identified autoantigen-specific $T$ cell responses associated with Th1 cytokine production of IL-2 and IFN- $\gamma(10)$. However, the role of antigenpresenting cells (APCs) in organ-specific T cell activation in this model has not yet been analyzed.

MHC class II molecules encounter and bind antigenic peptides as class II-peptide complexes on the cell sur-

Received for publication November 19, 2001, and accepted in revised form June 17, 2002.

Address correspondence to: Yoshio Hayashi, Department of Pathology, Tokushima University School of Dentistry, 3 Kuramotocho, Tokushima 770-8504, Japan. Phone: 81-88-633-7327; Fax: 81-88-633-7327;

E-mail: hayashi@dent.tokushima-u.ac.jp.

Conflict of interest: No conflict of interest has been declared.

Nonstandard abbreviations used: Sjögren syndrome (SS); thymectomized 3 days after birth (3d-Tx); nonthymectomized (non-Tx); antigen-presenting cell (APC); invariant chain (Ii); lymph node cell (LNC); ovalbumin (OVA); concanabalin A (ConA); mouse salivary gland (MSG); propidium iodide (PI). face of APCs for recognition by CD4 ${ }^{+} \mathrm{T}$ cells (11-13). The molecular mechanisms leading to formation of class II-peptide complexes and presentation of antigen on the cell surface begin with synthesis of class II $\alpha \beta$ heterodimers in the endoplasmic reticulum. These class II $\alpha \beta$ heterodimers associate early during biosynthesis with a type II membrane protein, the invariant chain (Ii) $(14,15)$. Inhibition of Ii degradation in B lymphoblastoid cells and murine spleen cells induces accumulation of class II-associated Ii fragments and inhibition of class II-peptide formation (16-19). Selective inhibition of the proteases responsible for both these degradative processes is a potential mechanism for modulating the immune response. Several lysosomal proteases have been implicated in the processing of Ii and antigenic peptides. Cathepsin B, the most abundant lysosomal cysteine protease, has been tied to Ii degradation using purified class II-Ii complexes (20). Cathepsin L, a potent cysteine-class endoprotease, is specifically inhibited by a fragment of the alternatively spliced Ii form p41 (21). Cathepsin S containing potent endoproteolytic activity is highly expressed in the spleen and professional APCs and other class II-positive cells and is inducible by IFN- $\gamma(22,23)$. In mouse splenocytes, inhibition of cathepsin $S$ also induces buildup of Ii breakdown products and attenuation of class II-peptide association, although the extent of this effect appears to be haplotype-dependent (24). We have developed specific inhibitors of cathepsin B (CA074), cathepsin L (Clik148), and cathepsin S (Clik60), in vivo as well as in vitro (25-27). Matsunaga et al. first reported that CA074 suppresses immune responses (28), suggesting that cysteine proteases in 
lysosomes play an important role in the functional differentiation of MHC class II-restricted $\mathrm{CD} 4^{+} \mathrm{T}$ cells. However, it is uncertain whether the inhibition of cathepsins B, L, and S blocks generation of the antigenic peptide on the development of autoimmune diseases. To address this important issue, antigen processing and presentation after specific inhibition of cathepsins were examined in a murine model for SS.

Studies presented here suggest that cathepsin $S$ plays an important role in processing of class II-Ii in autoantigen-presenting cells to generate class II molecules competent for binding antigenic peptide, and that inhibition of cathepsin S has important functional consequences in modulating the autoimmune response.

\section{Methods}

Mice. Female NFS/N-strain mice carrying the mutant gene sld (29) were reared in our specific pathogen-free mouse colony, and given food and water ad libitum. Thymectomy was performed on day 3 after birth (3d-Tx) in NFS/sld mice. Nonthymectomied (non-Tx) NFS/sld mice and C56BL/6 mice purchased from Charles River Japan Inc. (Atsugi, Japan) were used as controls.

Measurement of endogenous cathepsin activities. Salivary glands, regional lymph nodes, and spleens from $3 \mathrm{~d}-\mathrm{Tx}$ NFS/sld SS model, non-Tx NFS/sld, and control C57BL/6 mice were used to assay for cathepsin B, L, and $\mathrm{S}$ activity. Lysosomes were isolated for the assay by gentle homogenization of samples using a Teflon homogenizer (Microtec Co. Ltd., Funabashi, Japan) pestle in $0.25 \mathrm{M}$ cold sucrose. The suspension was centrifuged at $3,500 \mathrm{~g}$ for 10 minutes at $4^{\circ} \mathrm{C}$. The supernatant was centrifuged at $25,000 \mathrm{~g}$ for 20 minutes at $4^{\circ} \mathrm{C}$. The resulting pellet was resuspended with $50 \mathrm{mM}$ acetate buffer ( $\mathrm{pH}$ 5.0). The suspension fluid was frozen and thawed three times to disrupt lysosomal membranes. After three cycles of freezing and thawing, the fluid was centrifuged and the supernatant was used as a mitochondria and lysosome fraction. Cathepsin activities were assayed with Z-Arg-Arg-methyl coumarylamide (Peptide Institute, Osaka, Japan) as substrate at $\mathrm{pH} 5.0$ for cathepsin B, with Z-Phe-Arg-methyl coumarylamide for cathepsin $\mathrm{L}$, and using the method described by Inubushi et al. (30) for cathepsin S. The reaction was initiated by addition of substrate $(10 \mu \mathrm{M}$ final concentration) after preincubation with the test compound for 3 minutes at $37^{\circ} \mathrm{C}$. The fluorescence of the liberated 7-amino-4-methylcoumarin was measured in a fluorescence spectrophotometer (Hitachi Science Systems Ltd., Hitachinaka, Japan). Emission at $460 \mathrm{~nm}$ was measured with excitation at $370 \mathrm{~nm}$.

Cathepsin inhibitors. Specific inhibitors for cathepsin B (CA074), cathepsin L (Clik148), and cathepsin S (Clik60) have been developed with the help of computer-graphic modeling based on the stereo-structure as described previously (25-27). The common fragment, $N$-(L-trans-carbamoyloxyrane-2-carbonyl)-phenylalanine-dimethylamide, is required for specific inhibition of cathepsin $\mathrm{L}$. Seven novel inhibitors of the cathepsin $\mathrm{L}$ inhibitor
Katunuma (Clik) specifically inhibited cathepsin L at a concentration of $10^{-7} \mathrm{M}$ in vitro, while almost no inhibition of cathepsins B, C, S, and K was observed. Four of the Cliks are stable and showed highly selective inhibition for hepatic cathepsin $\mathrm{L}$ in vivo. One of the Clik inhibitors contains an aldehyde group and specifically inhibits cathepsin $\mathrm{S}$ at $10^{-7} \mathrm{M}$ in vitro (27).

In vivo treatment with cathepsin inhibitors. We examined the in vivo therapeutic effects of cathepsin $\mathrm{S}$ inhibitor (Clik60), cathepsin B inhibitor (CA074), and cathepsin L inhibitor (Clik148) in a murine model for SS. Each inhibitor (Clik60, CA074, and Clik148), dissolved in PBS, was administered intraperitoneally into model mice at doses of $0.1 \mathrm{mg} /$ mouse/day from 4 weeks to 7 weeks ( $n=10$ for each), and then analyzed at 8 weeks, compared with untreated SS model mice $(n=7)$.

Histology. All organs were removed from the mice, fixed with $4 \%$ phosphate-buffered formaldehyde $(\mathrm{pH}$ 7.2), and prepared for histologic examination. The sections were stained with hematoxylin and eosin. Histological grading of the inflammatory lesions was done according to the method proposed by White and Casarett (31). These slides were scored by three independent, pathologists in a blinded manner.

Proliferation assay. Single-cell suspensions of spleen cells or regional lymph node cells (LNCs) from 3d-Tx, non-Tx NFS/sld, and C57BL/6 mice were cultured in 96-well flat-bottom microtiter plates (Nalge Nunc Intl. Co., Rochester, New York, USA) in RPMI-1640 containing $10 \%$ FCS, penicillin/streptomycin, and $\beta$-mercaptoethanol. For proliferation assay, a total of $5 \times 10^{5}$ cells per well were cultured for 72 hours under stimulation of recombinant $\alpha$-fodrin protein (JS-1, 10 $\mu \mathrm{g} / \mathrm{ml})(10)$, ovalbumin (OVA) $(10 \mu \mathrm{g} / \mathrm{ml})$, and concanabalin A (ConA) $(5 \mu \mathrm{g} / \mathrm{ml})$, and pulsed with 1 $\mu \mathrm{Ci} /$ well of $\left[{ }^{3} \mathrm{H}\right]$ thymidine (NEN Life Science Products Inc., Boston, Massachusetts, USA) during the final 20 hours of the culture. $\left[{ }^{3} \mathrm{H}\right]$ thymidine incorporation was evaluated using an automated $\beta$ liquid scintillation counter. We further examined the in vitro preventive effects of cathepsin inhibitors $\left(10^{-7}\right.$ to $10^{-4} \mathrm{M}$ CA074, Clik148, and Clik60) for antigen-specific proliferative $\mathrm{T}$ cell responses. $\mathrm{T}$ cell purification was done using CD4 mAb-bounded immunomagnetic beads (Dynal, Oslo, Norway).

Flow cytometric analysis. Spleen cells and regional LNCs from 3d-Tx and non-Tx NFS/sld mice were analyzed by flow cytometry. Single-cell suspensions were stained with antibodies conjugated to phycoerythrin (antiCD4; Cedarlane Laboratories Ltd., Hornby, Ontario, Canada) and FITC (anti-CD8 from Cedarlane Laboratories Ltd.; anti-CD44, anti-CD45RB, anti-Mel-14, and anti-I-A ${ }^{q}$ from Pharmingen, San Diego, California, USA), and analyzed with an EPICS (Beckman Coulter, Miami, Florida, USA). Apoptotic or necrotic cells were detected by flow cytometry with an EPICS (Beckman Coulter) using the Annexin V-FITC Apoptosis Detection Kit (Genzyme Pharmaceuticals Co. Ltd., Cambridge, Massachusetts, USA). Viable cells $\left(1 \times 10^{5}\right)$ were 
gated according to size and scatter to eliminate dead cells and debris from analysis.

Measurement offluid secretion. Detection of tear and saliva volume of the treated and untreated SS animal model of NFS/sld mice was done according to a method as described (32). Five mice in each group were analyzed.

Measurement of cytokine production. Cytokine production from spleen cells of 3d-Tx and non-Tx NFS/sld mice was tested by two-step sandwich ELISA using a mouse IL-2, IL-4, and IFN- $\gamma$ kit (Genzyme Pharmaceuticals). In brief, culture supernatants from spleen cells activated with immobilized anti-CD3 mAb (10 $\mu \mathrm{g} / \mathrm{ml}$ ) (Cedarlane Laboratories Ltd.) for 3 days were added to microtiter plates precoated with anti-IL-2, $-\mathrm{IL}-4$, and -IFN- $\gamma$ capture antibody and incubated overnight at $4^{\circ} \mathrm{C}$. After addition of biotinylated detecting antibody and incubation at room temperature for 45 minutes, avidin-peroxidase was added and incubated at room temperature for 30 minutes. Plates were washed extensively with $1 \%$ Tween in PBS between each step. Finally, 2,2'-azino-di-3-ethylbenzthiazoline sulfonate (ABTS) substrate containing $\mathrm{H}_{2} \mathrm{O}_{2}$ was added and the colorimetric reaction was read at an absorbance of $450 \mathrm{~nm}$ using an automatic microplate reader (BioRad Laboratories Inc., Hercules, California, USA). The concentrations (in pg/ml) of IL-2, IL-4, and IFN- $\gamma$ were calculated according to the standard curves produced by various concentrations of recombinant cytokines.

Primary culture of mouse salivary gland cells. Mouse salivary gland (MSG) epithelial cells were prepared as previously described (33). Briefly, MSGs were minced into $1-\mathrm{mm}^{2}$ pieces, washed with HBSS without $\mathrm{Ca}^{2+}$ and $\mathrm{Mg}^{2+}$, and placed in a 60-mm dish containing HBSS with $0.76 \mu \mathrm{g} / \mathrm{ml}$ EDTA, $4.9 \mu \mathrm{g} / \mathrm{ml}$ L-ascorbic acid, and $4.9 \mu \mathrm{g} / \mathrm{ml}$ reduced glutathione. Fragments were washed with DMEM/STI and placed in a mixture of collagenase (type I, $75 \mathrm{U} / \mathrm{ml}$ ) and hyaluronidase (type $\mathrm{IV}, 500 \mathrm{U} / \mathrm{ml}$ ) dissolved in DMEM/F12 containing $10 \%$ FBS. The first digest suspension was passed through sterile $100-\mu \mathrm{m}$ nylon mesh filter and were redigested for 30 minutes by the same digestion procedure, and then the digest suspension was passed through a $100-\mu \mathrm{m}$ nylon mesh filter. Adherent cells, after culture in MEM containing 10\% FBS for 24 hours at $37^{\circ} \mathrm{C}$, were isolated as salivary gland epithelial cells. We confirmed that the cells over $95 \%$ were positively stained with anti-keratin polyclonal antibody.

Detection of serum autoantibodies against $120-k D a$ $\alpha$-fodrin. Serum autoantibody production against $120-$ $\mathrm{kDa} \alpha$-fodrin was analyzed by immunoblotting and ELISA as described previously $(6,7,10)$.

Confocal immunofluorescence analysis. IFN- $\gamma$-stimulated and nonstimulated MSG cells were fixed with 1\% paraformaldehyde and were incubated with $\mathrm{mAb}$ to $\mathrm{I}^{\mathrm{A}} \mathrm{A}^{q}$ molecule (Pharmingen) and FITC-labeled $\mathrm{AFN}_{303-318}$ peptide (described below). In vitro effects of cathepsin inhibitors were examined by the preincubation with each inhibitor $\left(10^{-5} \mathrm{M}\right)$ for $6-24$ hours. The labeled second antibody was Texas red-conjugated goat anti-mouse IgG (Molecular Probes Inc., Eugene, Oregon, USA). For microscopy, a Leica TCS-NT laser scanning microscope (Leica Microsystems Nussloch $\mathrm{GmbH}$, Nussloch, Germany) was used.

\section{Results}

Endogenous activities of cathepsins $B, L$, and $S$ in tissue samples. We first investigated endogenous activities of cathepsin S, cathepsin L, and cathepsin B in tissue samples of the salivary glands, regional lymph nodes, and spleens from SS model mice and controls. As shown in Figure 1, the activities of both cathepsin $S$ and cathepsin $L$ were significantly higher in the salivary glands, lymph nodes, and spleens from model mice than those of controls. No difference in activities of cathepsin B was found in any organ from these mice. Significantly inhibitory effects of cathepsin S inhibitor (Clik60), cathepsin L inhibitor (Clik148), and cathepsin B inhibitor (CA074) were observed in each organ in a dose-dependent manner. Moreover, we confirmed that cathepsin S activity in each organ was clearly inhibited by Clik60, but not by Clik148 and CA074 (data not shown).

Inhibitory effect of a specific inhibitor of cathepsin S (Clik60) on proliferative $T$ cell response to autoantigen. To address the role of autoantigen-reactive $T$ cells, we examined the proliferative $\mathrm{T}$ cell responses in the LNCs and spleen cells from model mice and controls. We found that the LNCs and spleen cells in SS model mice, but not in non-Tx NFS/sld and C57BL/ 6 control mice, at 8 weeks of age showed a significant increase in autoantigen-specific (JS-1-specific) $\mathrm{T}$ cell proliferation (Figure 2a; data of the spleen cells not shown). No significant differences were observed in the proliferative response stimulated with OVA $(10 \mu \mathrm{g} / \mathrm{ml})$ and ConA $(5 \mu \mathrm{g} / \mathrm{ml})$ among these mice. We examined the inhibitory effects of cathepsin inhibitors against autoantigen-specific $\mathrm{T}$ cell responses in vitro. In regional LNCs, but not in spleens, from model mice, a significantly inhibitory effect of Clik60 was observed in a dose-dependent manner (Figure $2 b$ ). In contrast, no inhibitory effects of Clik148 and CA074 were found. Annexin V/propidium iodide (PI) flow cytometric analysis revealed a small proportion of early apoptotic cells (annexin $V$-positive, PI-negative) and necrotic cells (annexin V-positive, PI-positive), indicating that Clik60 is not toxic and favors $\mathrm{T}$ cell survival in the LNCs (Figure 2c). Indeed, the Clik60 inhibitors at $10^{-7}$ to $10^{-4} \mathrm{M}$ concentrations do not block $\mathrm{T}$ cell proliferation to ConA (Figure 2d). These findings indicated that the dose of Clik60, $10^{-5}$ to $10^{-4} \mathrm{M}$, was sufficient to inactivate the autoantigenspecific $\mathrm{T}$ cell responses in vitro.

In vivo administration of cathepsin inbibitors. We next examined the in vivo therapeutic effects of Clik60, Clik148, and CA074 in a murine model for SS. Interestingly, the treatment with intraperitoneal injection of Clik60 $(0.1 \mathrm{mg} / \mathrm{mouse} /$ day $)$ was effective in preventing the development of autoimmune lesions in the lacrimal $(P<0.01)$, parotid $(P<0.05)$, and submandibular 

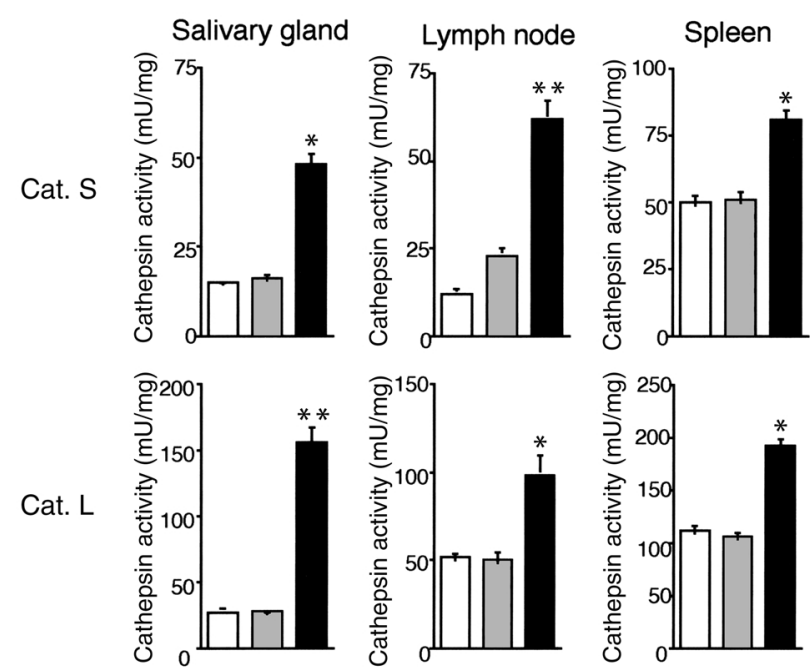

\section{Cat. B}
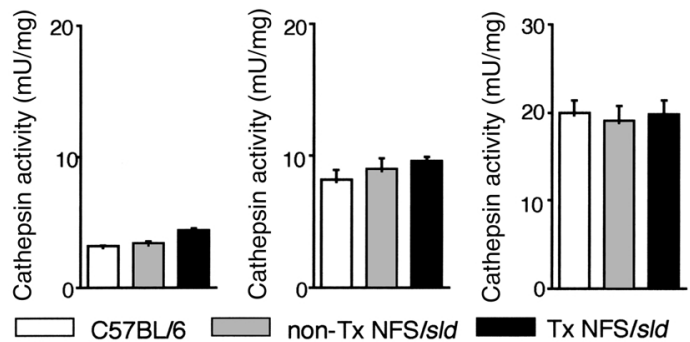

$(P<0.05)$ glands of the SS model mice, but not in those of groups injected with CA074 and Clik148 (Figure 3a). Representative histological features in the salivary and lacrimal glands are shown in Figure 3b. Moreover, the average saliva and tear volumes of Clik60-treated model mice were significantly higher than those of the untreated SS model mice (Figure 3c). The activation markers (CD44 ${ }^{\text {high }}, \mathrm{CD}^{4} \mathrm{RB}^{\text {low }}$, Mel-14 $\left.4^{\text {low }}\right)$ were clearly downregulated in LNCs gated on CD4 from Clik60treated model mice (Figure 4a). In addition, autoantigen-specific (JS-1-specific) T cell response was significantly inhibited in LNCs from Clik60-treated mice (Figure 4b). Culture supernatants from anti-CD3 $\mathrm{mAb}$-stimulated splenic T cells obtained from Clik60treated mice, but not those obtained from non-Tx

\section{Figure 2}

(a) Detection of proliferative T cell response of LNCs from SS model mice to organ-specific autoantigen (JS- 1$)\left({ }^{*} P<0.001\right.$, Student's $t$ test). No differences were found in OVA $(10 \mu \mathrm{g} / \mathrm{ml})$ and ConA $(5 \mu \mathrm{g} / \mathrm{ml})$ responsiveness. (b) In vitro preventive effect of proliferative $T$ cell response of LNCs, but not spleen cells, to JS-1 by Clik60 in a dosedependent manner. No inhibitory effects of Clik148 and CA074 were found. Data are expressed as stimulation indices \pm SEM. Three experiments from each group were performed at 8 weeks of age, and the mean values of index were statistically significant at ${ }^{*} P<0.05$ and ** $P<0.01$ (Student's $t$ test). (c) Annexin V/propidium iodide (PI) flow cytometric analysis revealed a small proportion of early apoptotic cells (annexin V-positive, $\mathrm{Pl}$-negative) and necrotic cells (annexin V-positive, $\mathrm{Pl}$-positive). (d) The inhibitors at $10^{-7}$ to $10^{-4} \mathrm{M}$ concentrations of Clik60 do not block T cell proliferation to Con A $(5 \mu \mathrm{g} / \mathrm{ml})$.

\section{Figure 1}

Endogenous cathepsin (Cat.) activities in tissue samples from SS model mice and controls. The activities of both cathepsin $\mathrm{S}$ and cathepsin $\mathrm{L}$ were significantly higher in the salivary glands, regional lymph nodes, and spleens from model mice than in those from controls $\left({ }^{*} P<0.05\right.$, ${ }^{*} P<0.01$, Student's $t$ test). Cathepsin activities were assayed as described in Methods.

NFS/sld mice, contained high levels of IL-4, but low levels of IL-2 and IFN- $\gamma$, by ELISA (Figure 5, a and b). Moreover, serum autoantibody production against 120$\mathrm{kDa} \alpha$-fodrin was exclusively inhibited in Clik60-treated mice, but not in other groups (Figure $5 \mathrm{c}$ ). A dilution curve, obtained by ELISA, illustrates that each case is capable of reacting with antigen (Figure $5 \mathrm{~d}$ ). These results strongly suggest that Clik60 plays an important role in preventing autoantigen presentation to generate class II molecules competent for binding antigenic peptide, resulting in inhibition of autoimmunity.

Possible role of MSG cells in autoantigen presentation. It has been well known that nonlymphoid cells that
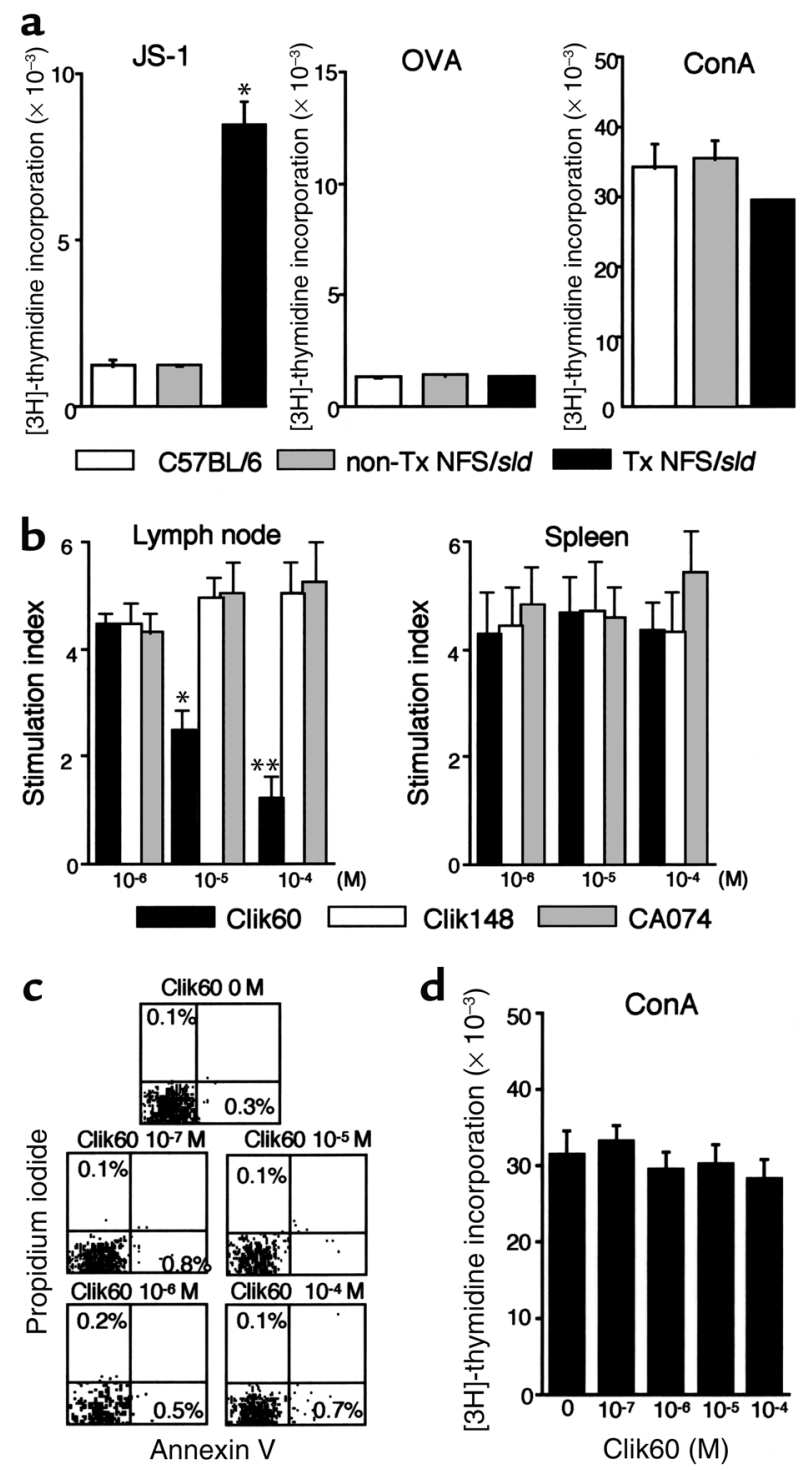
express MHC class II molecules provoke autoimmune responses in combination with costimulation $(34,35)$. To determine whether specific inhibition of cathepsin $S$ activity can functionally alter antigen presentation, primary cultured MSG cells, regional LNCs, and spleen cells from model mice and non-Tx control mice were compared in terms of their capacity to express class II molecule, and to react with organ-specific autoantigen as determined by flow cytometry and proliferation assay. Among these cells, a large proportion of class II-expressing (I-A ${ }^{q}$-expressing) cells was observed on MSG cells compared with spleen cells and LNCs from SS model mice (Figure 6a). Moreover, class II molecule can be stably induced by IFN- $\gamma$ stimulation on MSG cells from non-Tx NFS/sld control mice by flow cytometry (Figure 6b). Indeed, purified $\mathrm{CD}^{+} \mathrm{T}$ cells from model mice are capable of responding to IFN- $\gamma$-stimulated MSG cells, but not with nonstimulated MSG cells (Figure 6c). Autoantigen-stimulated (JS-1-stimulated) T cell response of LNCs using MSG cells was significantly inhibited by incubation with Clik60, but not incubation with Clik148 and CA074 (Figure 6d). These results suggest that MSG cells may function, at least in part, as autoantigen-presenting cells in the development of murine SS, and that inhibition of cathepsin S prevents both autoantigen presentation in the salivary gland cells and autoantigenspecific $T$ cell proliferation.

Inbibition of cathepsin S prevents class II-peptide association. We confirmed that activity of cathepsin S in MSG cells was clearly inhibited by Clik60 $\left(10^{-5} \mathrm{M}\right)$ (Figure 7a). Surface expression of class II molecule induced by IFN- $\gamma$ stimulation in MSG cells from non-Tx NFS/sld mice was clearly inhibited by incubation with Clik60, but not incubation with Clik148 and CA074, as determined by flow cytometry (Figure $7 \mathrm{~b}$ ). MHC class II molecules are synthesized with their peptide-binding site blocked by Ii, and they acquire the capacity to bind antigens only after Ii has been degraded in the compartments. Cathepsin S is known to play a pivotal role in proteolytic degradation of the Ii as a prerequisite for peptide binding to class II molecules (36). Recently, we have identified the pathogenic $T$ cell epitope peptide $\left(\mathrm{AFN}_{303-318}\right)$ on $\alpha$-fodrin autoantigen involved in autoimmune responses in this model (unpublished data). We observed colocalization of FITC-labeled $\mathrm{AFN}_{303-318}$ peptide and class II (I-A $\left.{ }^{q}\right)$ molecule in the IFN- $\gamma$-stimulated MSG cells (Figure 7c), suggesting that MSG cells may play a role in presenting autopeptide in the autoimmune responses. Moreover, confocal immunofluorescence analysis confirmed that inhibition of cathepsin $S$ prevents the surface expression of peptide-class II complex formation, while inhibition of cathepsin L and cathepsin B did not effect expression of peptide-bound class II molecules (Figure 7c).

\section{Discussion}

Therapeutic strategies for modulating the immune response in autoimmune disorders have centered on altering generation of the effector molecules that mediate the inflammatory reactions, e.g., corticosteroids, cyclosporin, and inhibitors of cytokines $(37,38)$. Our experiments demonstrate that selective inhibition of cysteine proteases is an additional potential strategy for modulating the autoantigen-derived immune response in class II-restricted disease processes. To our knowledge, this manuscript presents the first evidence that selective inhibition of cysteine protease cathepsin $\mathrm{S}$ in vivo has important functional consequences for MHC class II-dependent immune responses in autoimmune disease.

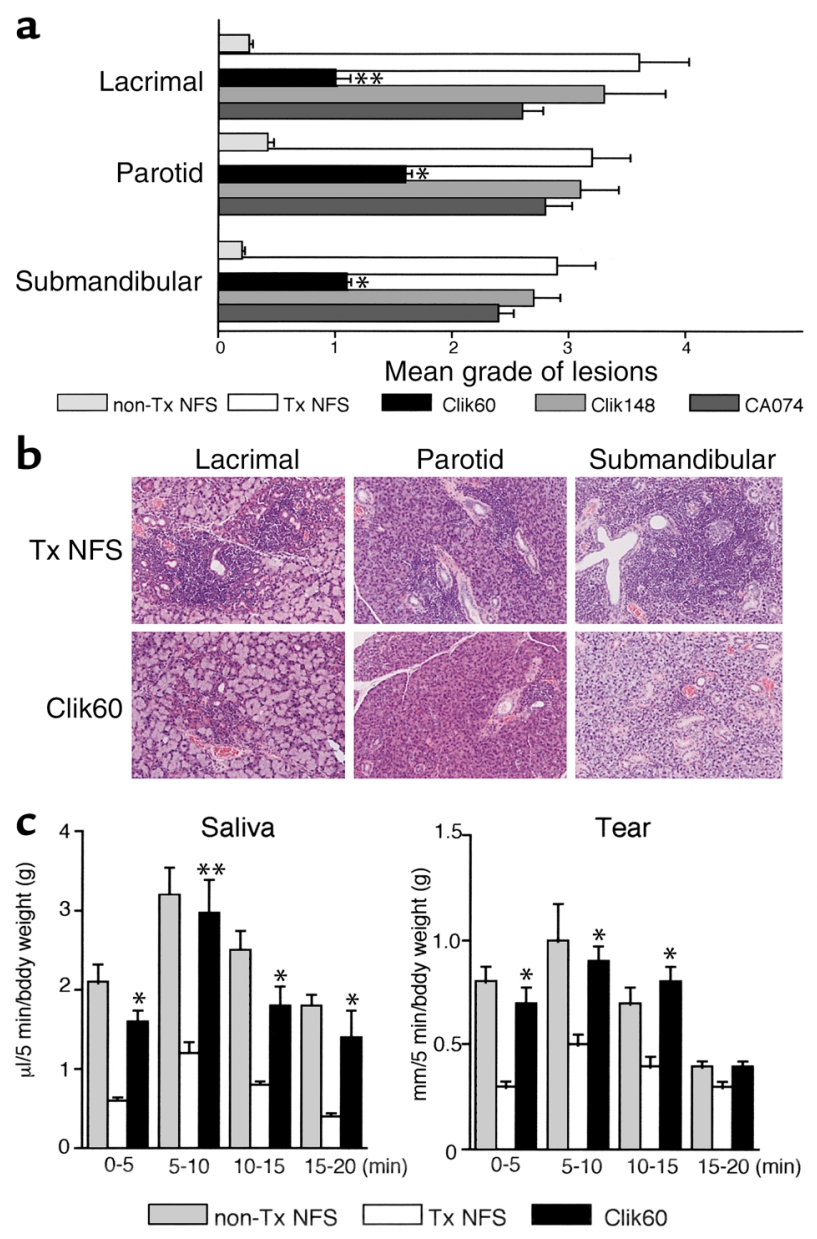

\section{Figure 3}

(a) Effects of in vivo administration of intraperitoneal injection of cathepsin inhibitors (Clik60, Clik148, and CA074). Each inhibitor was administered into SS model mice ( $n=10$ for each) at doses of $0.1 \mathrm{mg} / \mathrm{mouse} /$ day from 4 weeks to 7 weeks, and the results were analyzed at 8 weeks compared with those in untreated SS model mice $(n=7)$. Treatment with intraperitoneal injection of Clik60, but not treatment with Clik 148 and CA074, was effective in preventing the development of autoimmune lesions in the lacrimal, parotid, and submandibular glands of the SS model mice $\left({ }^{*} P<0.01\right.$, ${ }^{*} P<0.005$, Student's $t$ test). (b) Representative histologic features in the salivary and lacrimal glands showing preventive effects in Clik60-treated mice at 8 weeks of age. (c) The average saliva and tear volume of the SS model mice treated with Clik60 was significantly higher than that of control mice. Results are expressed as mean \pm SEM in five mice examined per group $\left({ }^{*} P<0.05,{ }^{*} P<0.005\right.$, Student's $t$ test). 
a
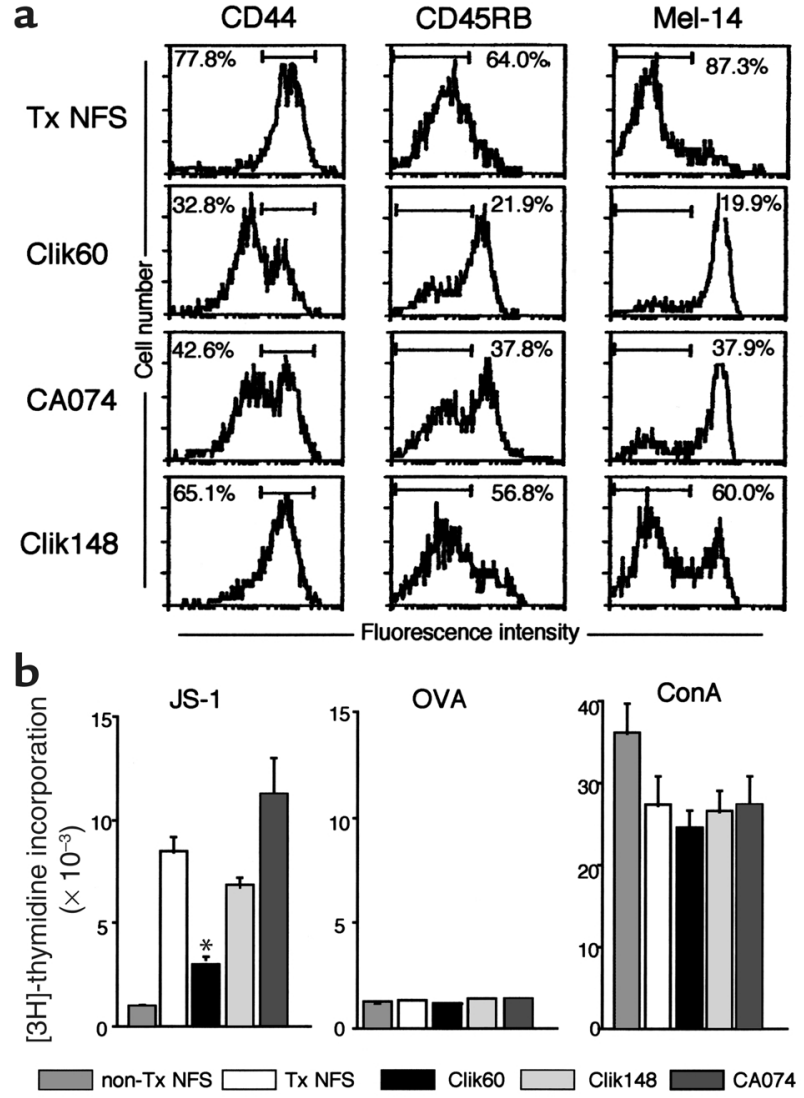

We reported previously a cleavage product of $120-\mathrm{kDa}$ $\alpha$-fodrin as an important autoantigen in the pathogenesis of SS in both an animal model and humans (10). The observation that thyrocytes express MHC class II molecules in Graves thyroiditis led Bottazzo and coworkers (39) to theorize that nonlymphoid cells that express MHC class II molecules provoke autoimmune responses by presenting autoantigens. Salivary gland epithelial cells from SS patients have been found to express MHC class II molecules in a large proportion of biopsy samples, and the number of positive cells generally increases with the number of lymphocytes infiltrating the gland (40-42). In the present experiments, a large proportion of class II-expressing (I-A ${ }^{q}$-expressing)

\section{Figure 5}

(a) Downregulation of Th1 cytokine production in SS model mice treated with Clik60. Culture supernatants from anti-CD3 $\mathrm{mAb}$-stimulated splenic T cells from Clik60-treated model mice ( 8 weeks old) contained a high level of IL-4, but low levels of IL-2 and IFN- $\gamma$, as measured by ELISA $\left({ }^{*} P<0.005,{ }^{*} P<0.001\right.$, Student's $t$ test). (b) No significant cytokine production in culture supernatants from anti-CD3 mAb-stimulated splenic $T$ cells from nonTx NFS/sld mice ( 8 weeks old). (c) Production of serum autoantibodies to $120-\mathrm{kDa} \alpha$-fodrin was diminshed in two mice (no. 1 and no. 2) treated with Clik60, as shown by immunoblotting. (d) A dilution curve illustrates that each case is capable of reacting with antigen. The same dilution of sera was examined in all cases by ELISA. OD492, optical density of $492 \mathrm{~nm}$.

\section{Figure 4}

(a) Upregulation of the activation markers (CD44high, CD45RB low, Mel-14low) in LNCs gated on CD4 from Clik60-treated mice, compared with control mice. (b) SS model mice treated with Clik60 showed a significant decrease of autoantigen-specific (JS-1-specific) T cell proliferation in LNCs, compared with controls $(P<0.01$, Student's $t$ test). Data are expressed as cpm per culture in triplicate.

cells was observed on MSG epithelial cells from SS model mice, and MHC class II molecule can be stably induced by IFN- $\gamma$ stimulation on MSG cells from syngeneic control mice. Moreover, autoantigen-stimulated (JS-1-stimulated) proliferative $\mathrm{T}$ cell response using MSG cells was clearly inhibited by the incubation with cathepsin S inhibitor (Clik60). In light of these results, it is possible that the salivary gland epithelial cells may function, at least in part, as autoantigen-presenting cells in the development of murine SS, and that inhibition of cathepsin $S$ prevents autoantigen presentation and subsequent peptide binding by class II molecules. MHC class II molecules bind a diverse array of peptides derived from the endocytic pathway and present them to $\mathrm{CD} 4^{+} \mathrm{T}$ cells. APCs have a pool of active class II mol-

a

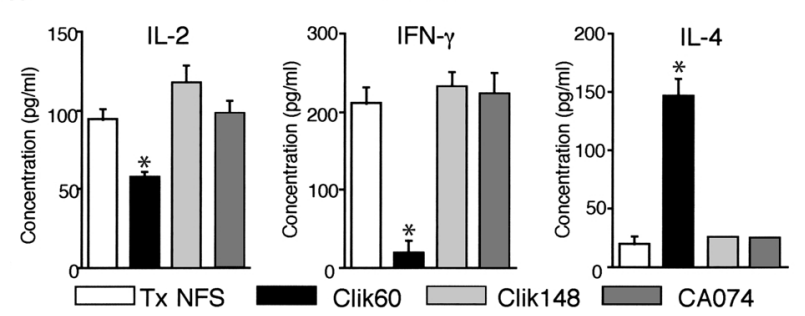

b

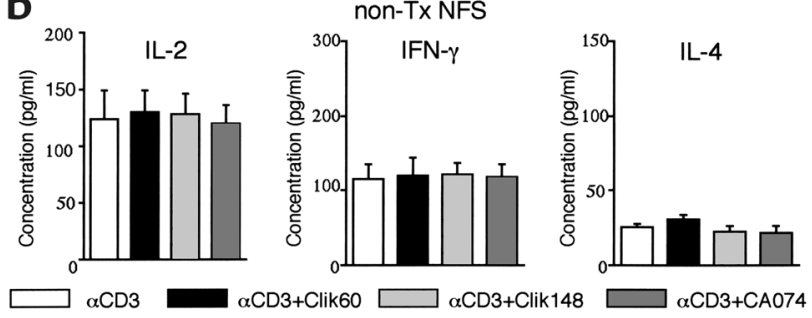

C
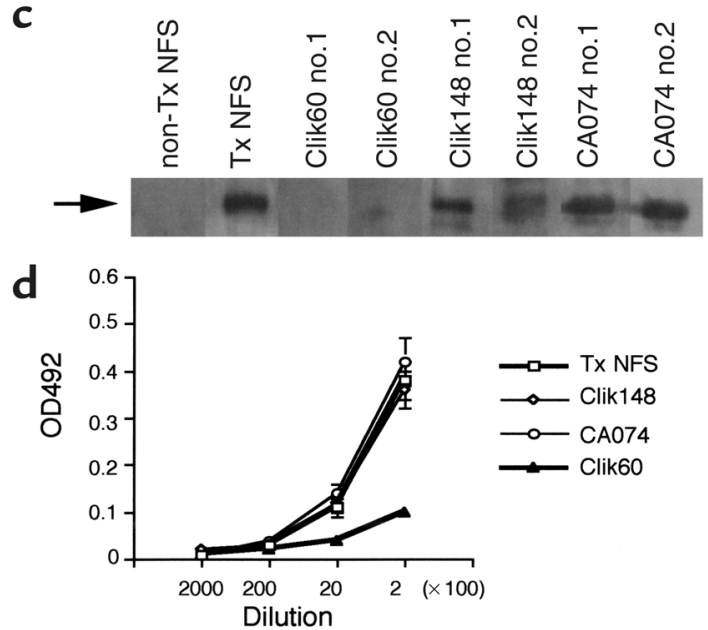
a

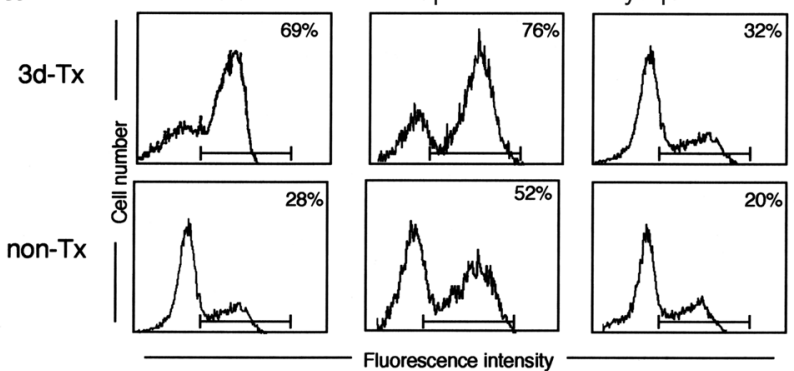

b

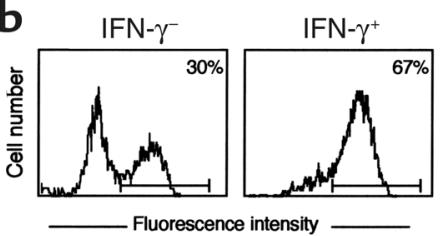

C

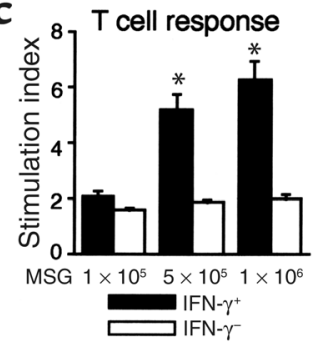

d
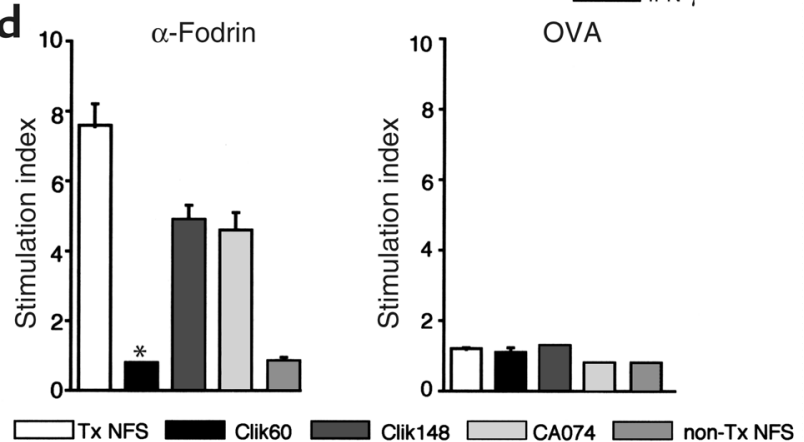

Figure 6

(a) A large proportion of class II-expressing (I-Aq-expressing) cells observed on MSG cells from SS model mice compared with those on spleen and LNCs, as determined by flow cytometry. (b) MHC class II molecule induced by IFN- $\gamma(100 \mathrm{U} / \mathrm{ml})$ stimulation in MSC cells from non-Tx NFS/sld control mice by flow cytometry. (c) Significant proliferative responses of purified CD4 $4^{+}$T cells $\left(2 \times 10^{5}\right)$ from model mice with IFN- $\gamma$-stimulated MSG cells $\left(5 \times 10^{5}\right.$ and $1 \times 10^{6},{ }^{*} P<0.01$, Student's $t$ test), but not from those with nonstimulated MSG cells. (d) Autoantigen-stimulated (JS-1-stimulated) proliferative T cell response of LNCs using MSG cells from SS model mice was significantly inhibited by the incubation with Clik60, but not the incubation with Clik148 and CA074 $\left({ }^{*} P<0.01\right.$, Student's $t$ test). Data are expressed as stimulation indices \pm SEM. Three experiments from each group were performed.

tion (49). In addition to cathepsin S and cathepsin L, cathepsin $\mathrm{F}$ has been recently implicated in Ii degradation and antigen presentation in alveolar macrophages (53). Thus, it would appear that different APCs utilize distinct cathepsins to mediate late-stage Ii degradation and to regulate MHC class II presentation. Moreover, cathepsin S-deficient mice showed diminished susceptibility to collagen-induced arthritis, suggesting a potential therapeutic target for regulation of immune responsiveness (50). In this study, the treatment with cathepsin $\mathrm{S}$ inhibitor was effective in preventing the development of autoimmune lesions in the salivary and lacrimal glands of the SS model mice. Almost entire remissions of SS were induced in the salivary and

ecules on their surface that can quickly load peptides from the extracellular milieu for $\mathrm{T}$ cell presentation (43). The kinetics of this $\mathrm{T}$ cell response may correlate with the kinetics of peptide binding to the surface class II MHC molecules (44-46).

Recent studies using cathepsin-deficient mice have shown that cathepsin D and cathepsin B are unnecessary for MHC class II presentation $(47,48)$, whereas cathepsin $\mathrm{S}$ and cathepsin $\mathrm{L}$ are important for antigen presentation by discrete populations of cells (49-52). Active cathepsin $\mathrm{S}$ was detected in B cells, dendritic cells, and peritoneal macrophages, where it was shown to be involved in the late stages of Ii degradation (50-52). By contrast, cathepsin L was detected only in macrophages and cortical thymic epithelial cells, where a defect resulted in severely impaired $\mathrm{CD}^{+} \mathrm{T}$ cell selec-

\section{Figure 7}

(a) Detection of cathepsin S activity in MSG cells and its inhibition by Clik60 $\left(10^{-5} \mathrm{M}\right)$. (b) Surface expression of class II molecule induced by IFN- $\gamma$ stimulation on MSG cells was clearly inhibited by the incubation with Clik60, but not the incubation with CA074 and Clik148, as determined by flow cytometry. (c) Colocalization of FITC-labeled $\mathrm{AFN}_{303-318}$ peptide and class II (I-A $\left.{ }^{q}\right)$ molecule in the IFN- $\gamma$-stimulated MSG cells. Prevention of the surface expression of peptide-class II complex formation was confirmed by the inhibition of Clik60, but not by inhibition of Clik148 and CA074.
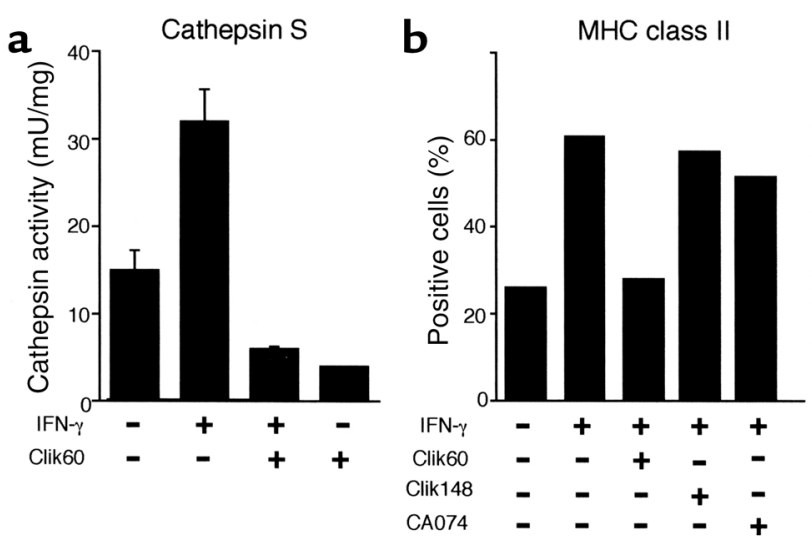

c

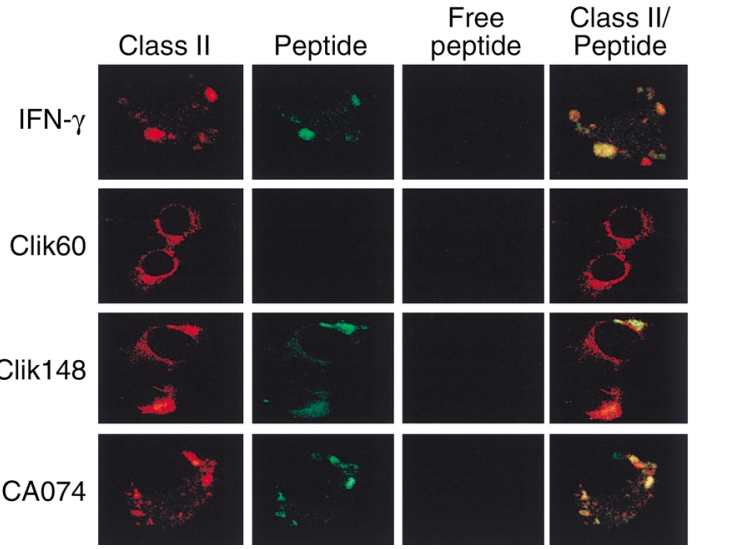


lacrimal glands by the Clik60 treatment. In addition, Clik60-treated mice showed a significant downregulation of autoantigen-specific (JS-1-specific) $\mathrm{T}$ cell response and Th1 cytokine expressions. Although we could not exclude the possibility that the Clik60 treatment in vivo inhibits only cathepsin S and that other enzymes could be involved, these results indicate that cathepsin S inhibitor plays an important role in preventing autoantigen presentation which is followed by inhibition of autoimmunity.

Ubiquitous expression and involvement in the terminal degradation of proteins that intersect the endocytic pathway were previously perceived to be the hallmarks of these proteinases. However, recent evidence has demonstrated that several cathepsins are expressed in a tissue-specific fashion and that partial proteolysis of specific biological targets is a key function of cathepsins in antigen processing. Analysis of cathepsin L-deficient mice revealed a profound defect in Ii degradation in thymic cortical epithelial cells but not in bone marrow-derived APCs. We have recently identified the pathogenic $\mathrm{T}$ cell epitope peptide $\left(\mathrm{AFN}_{303-318}\right)$ on $\alpha$-fodrin autoantigen involved in organ-specific autoimmune responses (unpublished data). We confirmed here that MSG cells may play an important role for presenting autopeptide in the autoimmune responses, and that inhibition of cathepsin $S$ prevents the surface expression of peptide-class II complex formation, while inhibition of cathepsin $\mathrm{L}$ and cathepsin $B$ did not. The differential expression of proteinases by distinct APCs may affect the types of peptides that are presented to $T$ cells and thereby the immune responses that are ultimately generated.

Taken together, our experiments support the hypothesis that cathepsin S, previously shown to be important in Ii processing in vitro, regulates MHC class II function and subsequent autoimmune responses in vivo. Thus, selective inhibition of cysteine protease cathepsin S may have important therapeutic potential in modulating class II-restricted autoimmune processes.

\section{Acknowledgments}

This work was supported in part by Grants-in-Aid for Scientific Research (12307040, 12557022) from the Ministry of Education, Science and Culture of Japan. K. Saegusa is a Research Fellow of the Japan Society for the Promotion of Science.

1. Fox, R.I., Robinson, C.A., Curd, J.G., Kozin, F., and Howell, F.V. 1986. Sjögren's syndrome. Proposed criteria for classification. Arthritis Rheum. 29:577-585.

2. Chan, E.K., Hamel, J.C., Buyon, J.P., and Tan, E.T. 1991. Molecular definition and sequence motifs of the $52-\mathrm{kD}$ component of human SS-A/Ro autoantigen. J. Clin. Invest. 87:68-76.

3. Kruize, A.A., Smeenk, R.J.T., and Kater, L. 1995. Diagnostic criteria and immunopathogenesis of Sjögren's syndrome: implications for therapy. Immunol. Today. 16:557-559.

4. Haneji, N., Hamano, H., Yanagi, K., and Hayashi, Y. 1994. A new animal model for primary Sjögren's syndrome in NFS/sld mutant mice. J. Immunol. 153:2769-2777.

5. Hayashi, Y., et al. 1996. Effector mechanism of experimental autoimmune sialadenitis in the mouse model for primary Sjögren's syndrome.
Cell. Immunol. 171:217-225.

6. Ishimaru, N., et al. 1999. Estrogen dediciency accelerates autoimmune exocrinopathy in murine Sjögren's syndrome through Fas-mediated apoptosis. Am. J. Pathol. 155:173-181.

7. Ishimaru, N., et al. 2000. Severe destructive autoimmune lesions with aging in murine Sjögren's syndrome through Fas-mediated apoptosis. Am. J. Pathol. 156:1557-1564.

8. Saegusa, K., et al. 2000. Mechanisms of neonatal tolerance induced in an animal model for primary Sjögren's syndrome by intravenous administration of autoantigen. Scand. J. Immunol. 52:264-270.

9. Saegusa, K., et al. 2000. Autoantigen-specific CD4 ${ }^{+} \mathrm{CD} 28^{\text {low }} \mathrm{T}$ cell subset prevents autoimmune exocrinopathy in murine Sjögren's syndrome. J. Immunol. 165:2251-2257.

10. Haneji, N., et al. 1997. Identification of $\alpha$-fodrin as a candidate autoantigen in primary Sjögren's syndrome. Science. 276:604-607.

11. Cresswell, P. 1994. Antigen presentation. Getting peptides onto MHC class II molecules. Curr. Biol. 4:541-543.

12. Germain, R.N. 1994. MHC-dependent antigen processing and peptide presentation: providing ligands for $\mathrm{T}$ lymphocyte activation. Cell. 76:287-299.

13. Wolf, P.R., and Ploegh, H.L. 1995. How MHC class II molecules acquire peptide cargo: biosynthesis and trafficking through the endocytic pathway. Annu. Rev. Cell Dev. Biol. 11:267-306.

14. Roche, P.A., Marks, M.S., and Cresswell, P. 1991. Formation of a ninesubunit complex by HLA class II glycoproteins and the invariant chain. Nature. 354:392-394.

15. Lamb, C., and Cresswell, P. 1992. Assembly and transport properties of invariant chain trimers and HLA-DR-invariant chain complexes. J. Immunol. 148:3478-3482.

16. Blum, J.S., and Cresswell, P. 1988. Role for intracellular proteases in the processing and transport of class II HLA antigens. Proc. Natl. Acad. Sci. USA. 85:3975-3979.

17. Nguyen, Q., Knapp, V., and Humphreys, R.E. 1988. Inhibition by leupeptin and antipain of the intracellular proteolysis of Ii. Hum. Immunol. 24:153-163.

18. Newcomb, J.R., and Cresswell, P. 1993. Structural analysis of proteolytic products of MHC class II-invariant chain complexes generated in vivo. J. Immunol. 151:4153-4163.

19. Neefjes, J.J., and Ploegh, H.L. 1992. Inhibition of endosomal proteolytic activity by leupeptin blocks surface expression of MHC class II molecules and their conversion to SDS resistant ab heterodimers in endosomes. EMBO J. 11:411-416.

20. Reyes, V.E., Lu, S., and Humphreys, R.E. 1991. Cathepsin B cleavage of Ii from MHC $\alpha$-and $\beta$-chains. J. Immunol. 146:3877-3880.

21. Bevec, T., Stoka, V., Pungercic, G., Dolenc, I., and Turk, V. 1996. Major histocompatibility complex class II-associated p41 invariant chain fragment is a strong inhibitor of lysosomal cathepsin L. J. Exp. Med. 183:1331-1338

22. Shi, G.P., Munger, J.S., Meara, J.P., Rich, D.H., and Chapman, H.A. 1992. Molecular cloning and expression of human alveolar macrophage cathepsin S, an elastinolytic cysteine protease. J. Biol. Chem. 267:7258-7262

23. Shi, G.P., et al. 1994. Human cathepsin S: chromosomal localization, gene structure, and tissue distribution. J. Biol. Chem. 269:11530-11536.

24. Villadangos, J.A., Riese, R.J., Peters, C., Chapman, H.A., and Ploegh, H.L. 1997. Degradation of mouse Ii: roles of cathepsins S and D and the influence of allelic polymorphism. J. Exp. Med. 186:549-560.

25. Towatari, T., et al. 1991. Novel epoxysuccinyl peptides: a selective inhibitor of cathepsin B, in vivo. FEBS Lett. 280:311-315.

26. Murata, M., et al. 1991. Novel epoxysuccinyl peptides:selective inhibitors of cathepsin B, in vitro. FEBS Lett. 280:307-310.

27. Katsunumua, N., et al. 1999. Structure based development of novel specific inhibitors for cathepsin $\mathrm{L}$ and cathepsin $\mathrm{S}$ in vivo and in vitro. FEBS Lett. 458:6-10.

28. Matsunaga, Y., Saibara, T., Kido, H., and Katunuma, N. 1993. Participation of cathepsin B in processing of antigen presentation to MHC class II. FEBS Lett. 324:325-330.

29. Hayashi, Y., Kojima, A., Hata, M., and Hirokawa, K. 1988. A new mutation involving the sublingual gland in NFS/N mice: partially arrested mucous cell differentiation. Am. J. Pathol. 132:187-191.

30. Inubushi, T., Kakegawa, H., Kishino, Y., and Katunuma, N. 1994. Specific assay method for the activities of cathepsin L-type cysteine proteinases. J. Biochem. (Tokyo). 116:282-284.

31. White, S.C., and Casarett, G.W. 1974. Induction of experimental autoallergic sialadenitis. J. Immunol. 112:178-185.

32. Delporte, B.C., et al. 1997. Increased fluid secretion after adenoviralmediated transfer of the aquaporin-1 cDNA to irradiated rat salivary glands. Proc. Natl. Acad. Sci. USA. 94:3268-3273.

33. Saito, I., et al. 1999. Fas Ligand-mediated exocrinopathy resembling Sjögren's syndrome in mice transgenic for IL-10. J. Immunol. 162:2488-2494. 
34. Londei, M., Lamb, J.R., Bottazzo, G.F., and Feldmann, M. 1984. Epithelial cell expressing aberrant MHC class II determinants can present antigen to cloned human T cells. Nature. 312:639-641.

35. Pujol-Borrell, R., et al. 1987. HLA class II induction in human islet cells by interferon- $\gamma$ plus tumor necrosis factor or lymphotoxin. Nature. 326:304-306.

36. Riese, R.J., et al. 1996. Essential role for cathepsin S in MHC class II-associated invariant chain processing and peptide loading. Immunity. 4:357-366.

37. Buttgereit, F., Burmester, G.-R., and Brand, M.D. 2000. Bioenergetics of immune functions: fundamental and therapeutic aspects. Immunol. Today. 21:192-199.

38. Balkwill, F., Foxwell, B., and Brennan, F. 2000. TNF is here to stay. Immunol. Today. 21:470-471.

39. Bottazzo, G.F., Pujol-Borrell, R., Hanafusa, T., and Feldmann, M. 1983. Role of aberrant HLA-DR expression and antigen presentation in induction of endocrine autoimmunity. Lancet. 2:1115-1119.

40. Fox, R.I., Bumol, R., Fantozzi, T., Bone, R., and Schreiber, R. 1986. Expression of histocompatibility antigen HLA-DR by salivary gland epithelial cells in Sjögren's syndrome. Arthritis Rheum. 29:1105-1111.

41. Franco, A., et al. 1987. Class II MHC antigen expression on epithelial cells of salivary glands from patients with Sjögren's syndrome. Clin. Exp. Rheumatol. 5:199-203.

42. Mircheff, A.K., et al. 1991. Class II antigen expression by lacrimal epithelial cells. An updated working hypothesis for antigen presentation by epithelial cells. Invest. Ophthalmol. Vis. Sci. 32:2302-2310.

43. Germain, R.N., and Hendrix, L.R. 1991. MHC class II structure, occupancy and surface expression determined by post-endoplasmic reticulum antigen binding. Nature. 353:134-139.
44. McConnell, H.M., Wada, H.G., Arimilli, S., Fok, K.S., and Nag, B. 1995. Stimulation of $\mathrm{T}$ cell by antigen-presenting cells is kinetically controlled by antigenic peptide binding to major histocompatibility complex class II molecules. Proc. Natl. Acad. Sci. USA. 92:2750-2754.

45. Beeson, C., et al. 1996. Early biochemical signals arise from low-affinity $\mathrm{T}$ cell receptor-ligand reactions at the cell-cell interface. J. Exp. Med. 184:777-782.

46. Rabinowitz, J.D., et al. 1997. Specific T cell recognition of kinetic isomers in the binding of peptide to class II MHC. Proc. Natl. Acad. Sci. USA. 94:8702-8707.

47. Villadangos, J.A., Riese, R.J., Peters, C., Chapman, H.A., and Ploegh, H.L. 1997. Degradation of mouse invariant chain: roles of cathepsins $S$ and $\mathrm{D}$ and the influence of major histocompatibility complex polymorphism. J. Exp. Med. 186:549-560.

48. Deussing, J., et al. 1998. Cathepsins B and D are dispensable for major histocompatibility complex class II-mediated antigen presentation. Proc. Natl. Acad. Sci. USA. 95:4516-4521.

49. Nakagawa, T., et al. 1998. Cathepsin L: critical role in Ii degradation and CD4 T cell selection in the thymus. Science. 280:450-453.

50. Nakagawa, T., et al. 1999. Impaired invariant chain degradation and antigen presentation and diminished collagen-induced arthritis in cathepsin S null mice. Immunity. 10:207-217.

51. Shi, G.P., et al. 1999. Cathepsin S required for normal MHC class II peptide loading and germinal center development. Immunity. 10:197-206.

52. Riese, R.J., et al. 1998. Cathepsin S activity regulates antigen presentation and immunity. J. Clin. Invest. 101:2351-2363.

53. Shi, G.P., et al. 2000. Role for cathepsin F in invariant chain processing and major histocompatibility complex class II peptide loading by macrophages. J. Exp. Med. 191:1177-1186. 